\title{
NOTE ON OSCILLATORY INTERFERENCE BANDS AND SOME PRACTICAL APPLICATIONS
}

\author{
By George O. Squier and Albert C. Crehore
}

In making measurements of any kind the observer usually requires that some form of mass shall be moved. The agencies employed to move this mass afford a convenient means of classifying instruments into four general groups according as the forces are applied:

I. Mechanically.

II. By Heat.

III. By the Magnetic Field.

IV. By the Electric Field.

These groups may be subdivided by taking account of the manner in which the matter to be moved is observed. The present inquiry chiefly concerns one method of noting this in an indirect way by observing the motion of interference bands or fringes. By this means the motion of the matter is derived and may be measured accurately if desired.

The marvelous beauty, sensibility, and accuracy of light interference phenomena are well known, but they are only fully appreciated by those very few specialists who devote their attention to these subjects. Such considerations have led the authors to devise some new types of instruments which are simple in construction, portable, and yet attain a sensibility at present only reached by laboratory apparatus. Instruments illustrative of each of the four classes above mentioned have been constructed.

Interference phenomena in light are many and varied in character, the principal examples being those produced by thin plates, including Newton's rings, thick plates, reflection by mirrors, diffraction, and that produced by two similar point sources, together with additional phenomena due to polarization. The instruments to be described make use of interference by thin 
plates and reflection by mirrors and opportunity has not been presented to examine other methods of interference applied to similar purposes.

\section{OSCILLATORY BANDS}

If a plate of glass is laid upon a second plate, which may be glass or any substance having a surface that will reflect light, it is well known that brilliant bands, being alternately light and dark in character, may be seen by reflection if the source of light is monochromatic. The geometrical character of the surfaces can be determined by observing the contour of the curves followed by these bands. Ordinary pieces of window glass produce these bands very well, but they are curved in various ways indicating that the surfaces are not plane. If the surfaces are optically plane, and the upper plate rests by its own weight upon the lower plate, the bands are usually formed in straight lines. The more nearly parallel to each other the planes become, the greater the distance between the bands. The two planes form a wedge with a very small acute angle, and the edge of this wedge, where the planes intersect, is a line parallel to the bands.

A pressure applied to some point of the upper plate, so as to press the two plates nearer together, produces two separate effects. It alters not only the angle of the wedge, but also rotates the edge through a considerable angle. These changes affect the bands each in its own way, the first broadening them and increasing the distance between adjacent bands; the second rotating the whole parallel system of bands by the same angle by which the edge of the wedge is rotated.

With large plates the air imprisoned between them retards any rapid motion of readjustment after a pressure is applied, but with plates of smaller area and mass, from 5 to ro millimeters in diameter, there is a quick response to a change of pressure, and the angular motion of the bands is proportionally greater for a given change. In using such plates in the instruments to be described the pressure between them is varied, and a consequent motion of the bands produced, an alternating force causing an oscillatory motion of the bands. 'The types of instruments therefore differ according to the means for varying the pressure. 


\section{PRESSURE MECHANICALLY APPLIED}

Fig. I shows an instrument for detecting or measuring small changes of pressure. A diaphragm, $\mathrm{D}$, is clamped between the solid metal pieces, $A$ and $B$, in such manner as to enclose a small air chamber, $C$, provided with an outlet, $T$. At the center of the disc is cemented a piece of plane optical glass, $\mathrm{P}_{1}$, and upon this a similar plate, $\mathrm{P}_{2}$, is laid, merely resting by its own weight. An observer looks into the instrument from above and sees the light from some convenient monochromatic source reflected from the two glass plates. The interference bands appear clear and distinct and are straight.

Any change of pressure of the air within the chamber, $C$, the atmospheric pressure without remaining constant, deflects the diaphragm and carries both plates together, either up or down, according to circumstances, but this alone will not change permanently the pressure between the glass plates, which is necessary to move the bands. If the

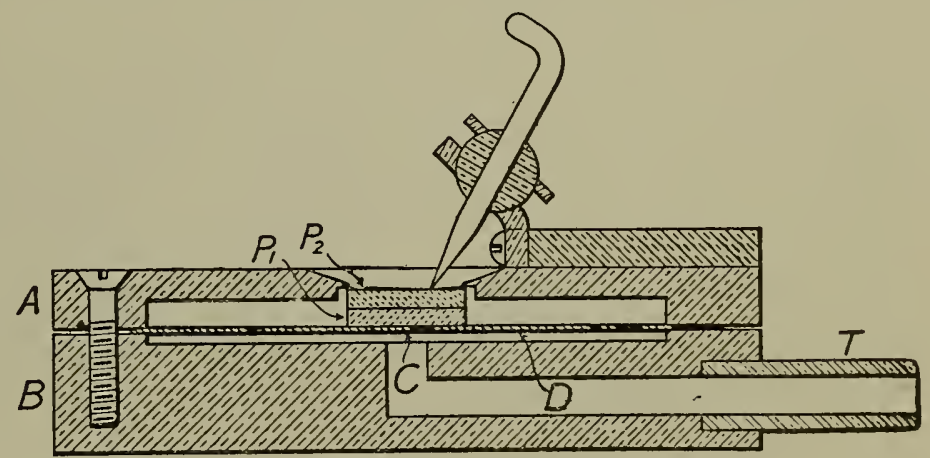

Fig. 1. Section of Interference Instrument for Varying the Pressure Mechanically. Designed for Physiological Measurements upper plate were held stationary with respect to the frame, A B, while the lower plate moved, this would move the bands. The object may be accomplished in a very simple manner by the use of a rigid steel rod or "pointer" attached to the frame and adjustable until it makes contact with the upper glass plate.

In adjusting this pointer the bands change to a new position at the first contact and remain as long as the pressure is the same. For a given setting of the pointer an increase of pressure in the chamber moves the diaphragm outward and increases the pressure between the glass plates. The use of a pointer localizes the pressure at one point of the upper plate and produces the changes above described. The bands broaden out, and their direction revolves through an angle dependent upon the amount of increase 
of pressure. If the air pressure is made to increase and decrease, the system of bands oscillates in phase with the pressure changes.

The pointer is so constructed in a ball and socket joint, the ball being split so that the pointer slides friction-tight through it, that it can be moved easily to any part of the surface of the upper glass plate, for it is found that there are critical points on the plate where the sensibility of the instrument is greatly increased. In exploring the plate with the pointer for an initial setting it has been found that particular areas are much more senstitive to minute changes in pressure than the remainder of the plate. It is easy to obtain an angular motion of 180 degrees for small changes in the adjustment of the pointer.

With this instrument a sufficient change of pressure of the air is obtained to oscillate the bands through a large angle by connecting it to the pulse at the wrist with an ordinary stethoscope and rubber tube as shown in Fig. 2. In this manner the character of the human heart action has been observed visually by the movement of the bands.

Fig. 3 shows two photographic cardiograms taken by means of the instrument shown in Fig. I. In addition, an ordinary camera having a plate holder adapted to move the plate past a slit and a plane glass mirror to turn the light reflected from the interference plates horizontally into the camera were used. A very convenient source of light is an ordinary commercial mercury-vapor lamp. It does not give pure monochromatic light, but the bands are very distinct, the predominant colors being violet and green. For photographic purposes it is very superior, and any rapid photographic plate gives good results.

\section{PRESSURE PRODUCED BY HEAT}

In the diagram Fig. 4 two plates of ordinary glass, $A B C D$ and EFGH, were used, the lower plate being first silvered over its whole surface and a portion, $\mathrm{AJB}$ and $\mathrm{CKD}$, removed. The silver remaining includes the narrow neck $\mathrm{JK}$ between the terminals $\mathrm{L}$ and $M$. The upper plate is secured by clamps so as to cover this neck, leaving an area for observing the interference bands between the plates. Bands appear throughout the entire extent of the upper plate, but are less brilliant over the silver. Upon passing 


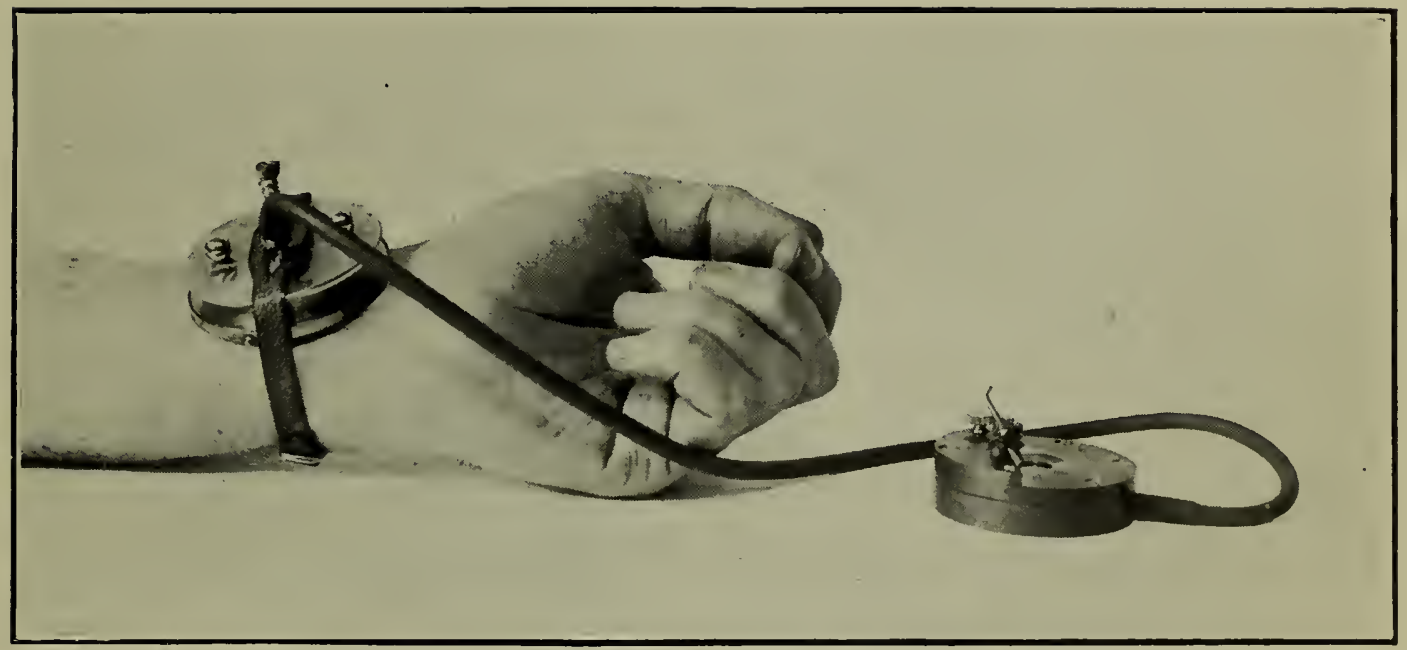

Fig. 2. Method of Connecting Instrument for Observing Heart Action 

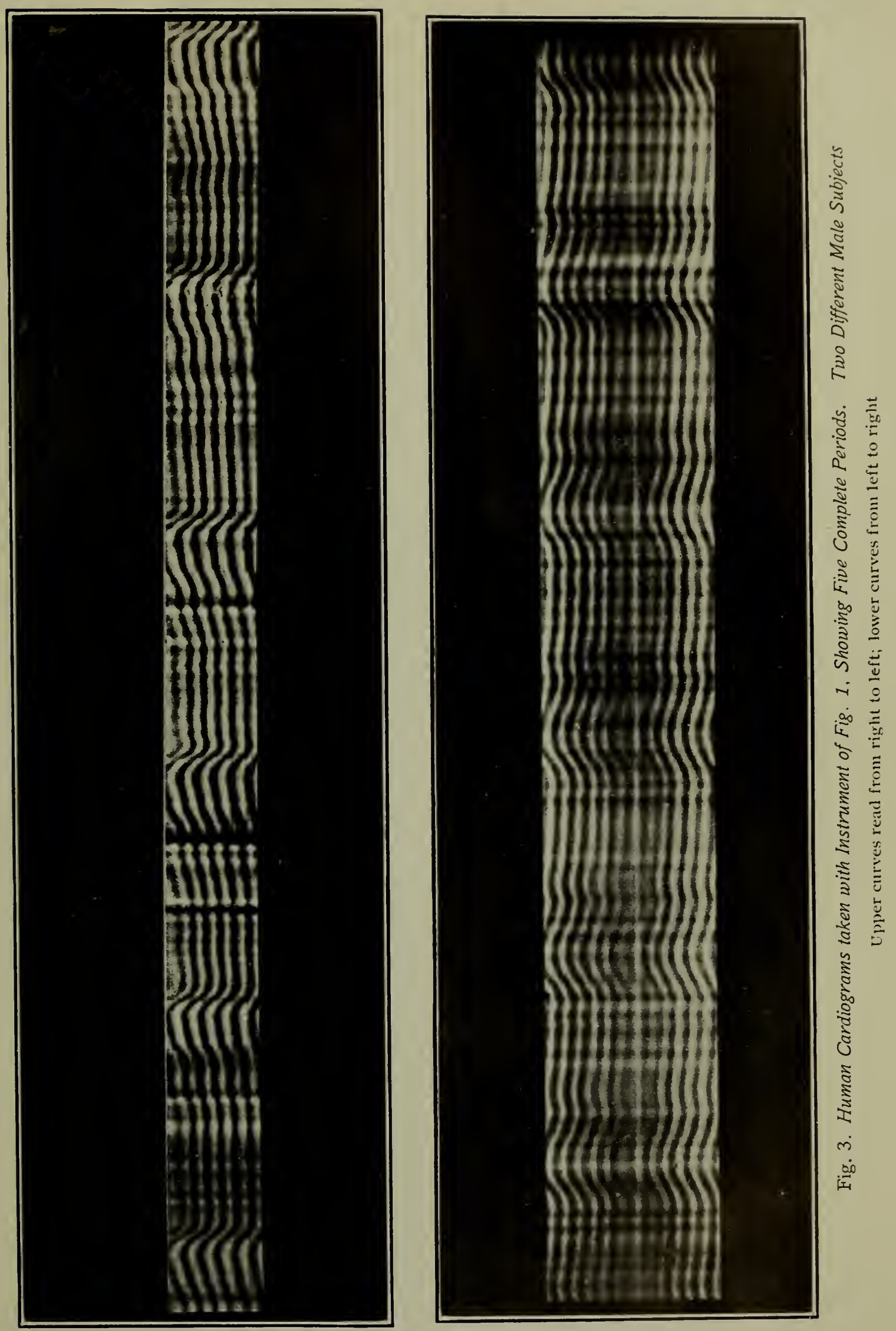
an electric current the heat developed is localized in the narrow neck at the center of the plates. At this point the glass of both plates expands, making a small elevation on each plate, forcing them apart and shifting the interference bands. In a first attempt at an instrument of this kind, with a resistance between terminals of 5 I ohms, it required as much as O.I 8 watts to cause an observable motion of the bands. Current 0.063 amperes and volts 2.9. Other instruments of similar construction were made having different resistances at the neck. With a resistance of $\mathrm{I}, 800 \mathrm{ohms}$,
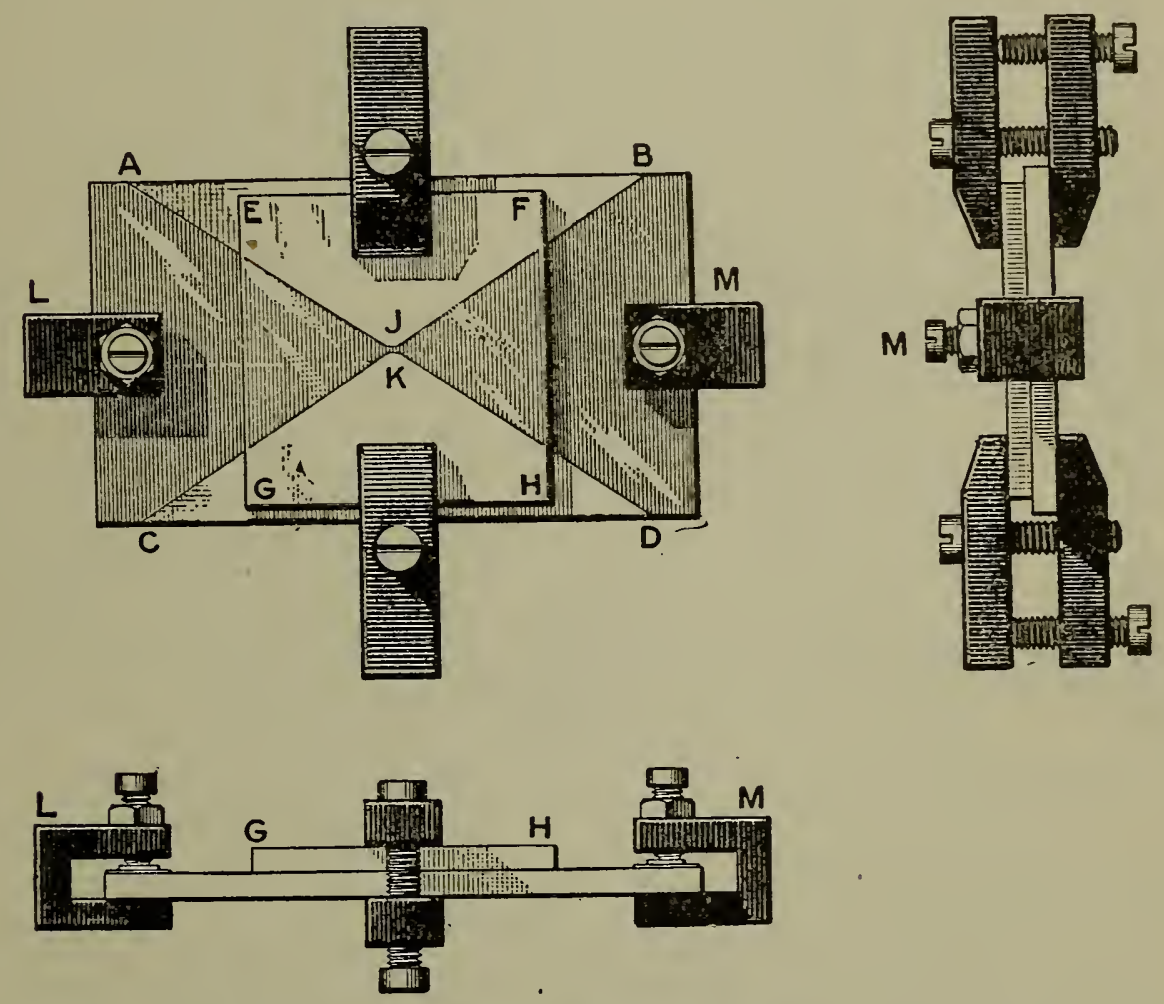

Fig. 4. Interference Instrument Operated by Heat. Silver Between Two Plates of Glass

o.I 6 watts were required to move the bands approximately the same as above. Current, 9 milliamperes.

Approximately the same number of watts is required to move the bands with widely different resistances. It thus appears that this amount of heat is a quantity imposed by the nature of silver and glass. The sensibility is limited by their coefficients of expansion. Hard rubber was tried as being a substance of large coefficient of expansion, but since it is opaque, the bands were observed 
from the opposite side of the silvered plate. They can only be seen over those portions of the glass where the silver is absent, and are not so brilliant with rubber as with glass, but yet sufficiently distinct to be used. The coefficient of expansion of hard rubber is 7 or 8 times that of glass. A motion of the bands was produced with 0.005 watts, current ro milliamperes, and resistance 50 ohms. Substituting gutta-percha for hard rubber increased the sensibility to o.0oI 4 watts.

Another form of heat instrument, Fig. 5, represents a glass plate silvered on the upper surface and provided with terminals as in Fig. 4. A piece of mica, as nearly plane as possible, 0.0075
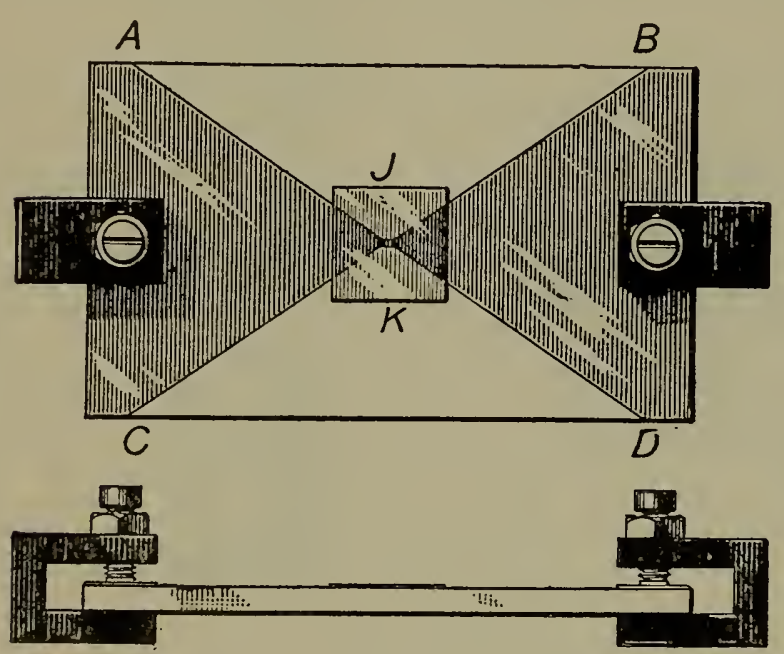

Fig. 5. Interference Instrument Operated by Heat. Silver Between Glass and Thin Mica Plates $\mathrm{mm}$ thick and I $\mathrm{cm}$ square is pressed against the glass so as to cover the neck. In this condition the mica adheres to the glass so that it is not easily displaced. The bands are brilliant, having various curved contours. With such plates there generally occur two kinds of critical points, one elliptical and the other hyperbolic in character. The points of contact are at the centers of elliptical areas, and the points of greatest separation are at the centers of two sets of curves hyperbolic in character. Any motion is most apparent at these points, and the sensibility is increased by moving the mica plate over the neck so as to bring one of these critical points into its neighborhood.

When the neck was cut across with a knife edge and powdered graphite substituted, the sensibility was as follows:

Resistance

Current

EMF

Power
I 29 ohms.

0.00093 amperes.

O. I 2 volts.

o.0OOII 3 watts. 
This increase in sensibility is due to the small mass of the mica plate which rested without clamps by its own weight upon the glass. The mica being easily flexed and not plane exhibits individual peculiarities. The time required for the bands to assume their final position is so brief that Morse signals may be read at a rapid rate in any of the forms of heat instruments described.

\section{PRESSURE VARIED BY THE MAGNETIC FIELD}

The optical apparatus for producing oscillatory bands is readily made operative by the magnetic field. A convenient method is to mount the glass plates upon an iron diaphragm acted upon by a magnetic field as in a telephone receiver. ${ }^{1}$ The order of magnitude of the sensibility obtained by using a telephone receiver in this manner is:

$\begin{array}{ll}\text { Resistance } & 620 \text { ohms. } \\ \text { Current } & 1.26 \times \mathrm{IO}^{-6} \text { amperes. } \\ \text { EMF } & 0.00077 \text { volts. } \\ \text { Power } & 9.8 \times \mathrm{IO}^{-10} \text { watts. }\end{array}$

A telephone used optically in this way is applicable to wireless telegraphy. Wireless messages have been received by merely removing the telephone now commonly used, and substituting the optical telephone in its place, without making any change whatever in the arrangement of the electrical circuits. Both the optical receiver and the telephone receiver have been used simultaneously by the same person, the two instruments being connected in circuit at the same time. This permits an observer to both see and hear a wireless message simultaneously. The probable advantages of two physiological senses to examine corroboratively and simultaneously a single phenomenon are worthy of careful investigation.

When no signal is being sent from the transmitting station, the bands remain at rest in a given fixed position. When a signal is received the bands rotate quickly to a new position depending upon the energy of the received signal and remain in the new position until the termination of the signal. Dots and dashes are thus easily read.

${ }^{1}$ Barus.: Am. J. Sci. (4) 3, 219; 1897. 
In adapting a telephone to vary the pressure by the magnetic field, it should be remembered that it is designed primarily for the ear, whereas what is here desired is maximum displacement for a minimum of applied energy. A marked increase of sensitiveness has been obtained by nothing more than a redistribution of the iron in the diaphragm. The ultimate sensibility of this galvanometer will only become known when its electro-magnetic system has received the same careful design as is now given to other galvanometers for special purposes.

It is frequently desirable to make measurements of alternating currents of low frequencies. Below 16 cycles nothing is audible in a telephone; but by this optical method the useful range of the telephone is extended to infra-sounds of very low frequencies such as are required by ocean cable telegraphy.

\section{USE OF CIRCULAR REFLECTION FRINGES FROM PLANE PARALLEL MIRRORS}

In the interference system heretofore described the glass plates are in contact, giving phenomena due to thin plates. A different arrangement has been used in which these plates are separated by several millimeters. The diagram, Fig. 6, shows a source of monochromatic light, $\mathrm{S}$, partially reflected from the glass plate, $\mathrm{G}$, placed at an angle of 45 degrees, down through the semitransparent mirror, $\mathrm{P}_{2}$, consisting of a glass plate thinly silvered on the lower side, to the lower plate $\mathrm{P}_{1}$, which is thickly silvered and polished on the upper side. Thence it is reflected perpendicularly upward, part of the light passing through the semi-transparent mirror, $\mathrm{P}_{2}$, and part being reflected again back to the plate $\mathrm{P}_{1}$. The former portion passes directly to the eye through the glass plate, $\mathrm{G}$, and the latter goes over the same course after reflection between the two plates. When these plates are adjusted to parallelism circular fringes are perceived by the observer at $\mathrm{E}$ looking downward into the plates $\mathrm{P}_{1}$ and $\mathrm{P}_{2}$. This arrangement is a modified form of the Fabry-Perot interferometer as employed by Priest. ${ }^{2}$

The lower plate, $\mathrm{P}_{1}$, is mounted upon the diaphragm, $\mathrm{D}$, of a telephone. The observer sees a system of well-defined concentric

\footnotetext{
2 This Bulletin, 5, p. IIo, on "A New Method of Determining the Focal Length of a Converging Lens."
} 
rings which expand or contract as the distance between the plates changes, the central spot becoming alternately light and dark.

If an alternating current of low frequency is applied, the circles visibly expand and contract alternately. As the frequency is increased the motion becomes so rapid that the whole field may appear uniformly illuminated, due to the persistence of vision. At 30 cycles some traces of motion are visible, but at 60 the field appears uniform if the current is sufficiently large.

When used as a telegraph receiver for alternating current, dots and dashes are read by noting the appearance and disappearance of the fringes. No apparent motion of the fringes, but merely their presence or absence in a EX fixed position, is seen by the unaided eye.

For low frequencies, four or five per second, such as are obtained in an ocean cable, the motion of the bands may either be followed by the eye or recorded photographically.

In using this method, external
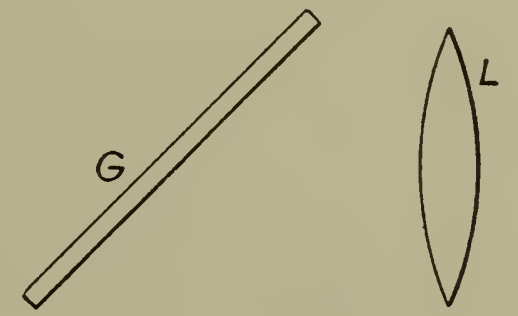
$\underset{x}{S}$ sounds or jars vibrate the diaphragm and disturb the fringes. Even talking in the room is easily seen. No trouble from this source is experienced with the oscillatory band system formed by plates in contact. On the other hand, the fact that the diaphragm is perfectly free to vibrate in the circular fringe svstem makes this form very sensitive to alternating currents.

\section{PRESSURE VARIED BY THE ELECTRIC FIELD}

The diagram, Fig. 7, shows a form of instrument for measuring changes of electromotive force. It is an electrostatic voltmeter, and may also be called an "optical condenser" in that the motion 
of the two condenser plates is observed visually as the electrostatic field between them is varied.

The flexible glass plates, A \& B, $25 \times 25 \times 0.125 \mathrm{~mm}$ are each silvered on one side, the silvered sides being turned toward each other. A piece of paper, $\mathrm{C}$, with a circular hole $2.5 \mathrm{~cm}$ in diameter separates the plates by a fixed distance, $0.05 \mathrm{~mm}$, and leaves the condenser with air between the plates at the central portion. The two optically plane plates, as before, are placed upon the upper glass plate, and a pointer
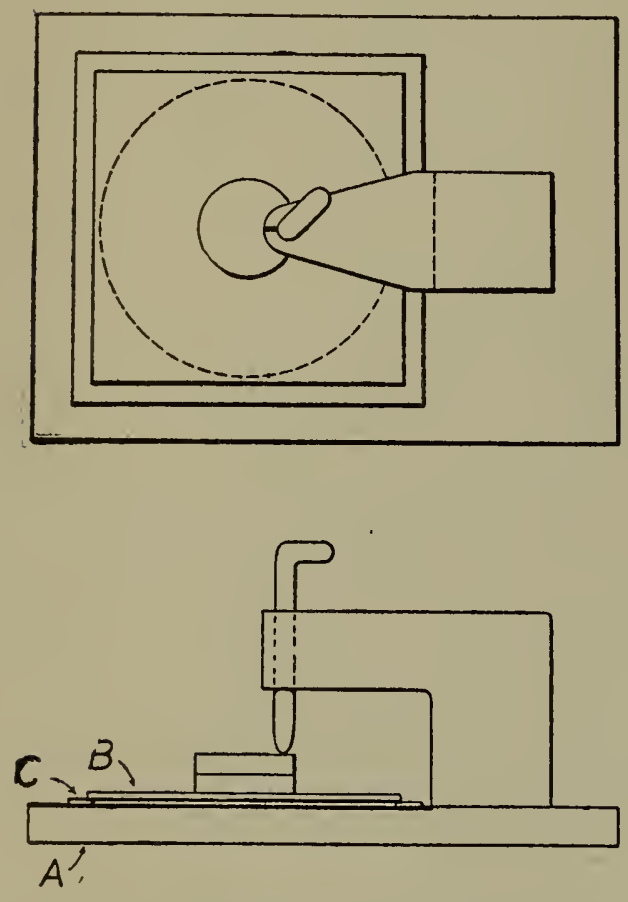

Fig. 7. Optical Condenser. Pressure Varied by the Electric Field. One Unit fixed to the frame holding the lower plate is brought to bear upon the upper plate. When the potential difference is changed from o to 20 volts an appreciable motion of the bands is observed. The volt-sensibility is increased, however, by an initial voltage which is slightly varied. A change from 106 to ro8 volts produced an appreciable motion of the bands. The sensibility being proportional to the difference between the squares of the electric fields, the change in the first case gives 400 as the difference of the squares, and in the second case 428 , values within the errors of observation. Although the sensibility is not high when applied to measuring small voltages, the energy required to produce a deflection is a small quantity. The computed capacity of this condenser is $0.000085 \mathrm{mf}$ and the energy o.108 ergs. This is roughly equal to the work of raising I milligram I millimeter.

\section{OPTICAL CONDENSER PILE}

Fig. 8 illustrates a pile of two condensers, one above the other, the upper carried on a small glass block, D, centrally located. The displacement of the top plate of the pile is amplified by this 
arrangement. The sensibility of the two condensers is practically twice that of one alone. A change of potential difference from o to Io volts gives an indication with the condensers in parallel. The extent to which condensers may be added in this manner to advaritage depends upon the mass of the moving parts.

This superposition of units is hardly possible in the electromagnetic form of instrument.

The volt-sensibility has been further increased by the following construction. A thick plane glass plate was silvered on the upper surface and one-half removed. A thin glass plate, $0.125 \mathrm{~mm}$, was prepared in the same manner. A thin coat of lacquer was baked on one of the silver surfaces, and the thin plate laid upon the thick one with the silvered portions superposed. Bands are observed over the unsilvered area, although there
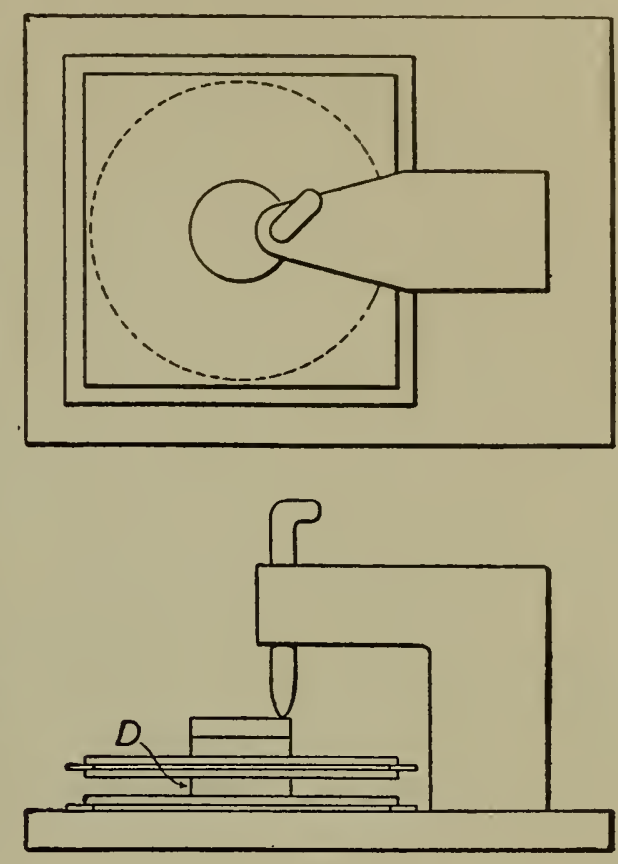

Fig. 8. Optical Condenser Pile. Pressure Varied by the Electric Field. Two Units were two coatings of silver and one of lacquer between the glass surfaces. The volt-sensibility is a change of potential difference from o to 3.4 volts.

\section{COMPARATIVE SENSITIVITIES OF EYE AND OF EAR INSTRUMENTS}

In using two of the physiological senses to observe phenomena, a comparison of their ultimate sensitivities is important. In making such a comparison, the frequency of vibration is a most important factor. The limits of sound frequencies which affect the ear makes this evident. These are of course not definite, but the lower is not far from 16 periods per second. At this limit the sensitivity to the eye is infinitely greater than to the ear. As the frequency is continuously increased, the sensitivity to the ear rises rapidly and the ratio falls. In the present state of our knowledge it is not known to what minimum value this ratio falls; but that there is a minimum at some particular frequency

$$
58397^{\circ}-\mathrm{II}-\mathrm{IO}
$$


seems probable, since it is definitely known that the sensitivity to the ear again approaches zero at an upper limit in the neighborhood of from 20000 to 40000 periods per second.

The energy required to produce sounds of a definite amplitude rises rapidly with increase of frequency, and the properties of the atmosphere at these high velocities are far different from those at low velocities. Some of the energy required to vibrate a diaphragm is absorbed within the substance of the diaphragm itself, but at these higher frequencies the larger portion is transmitted to the atmosphere. By placing the diaphragm in vacuo, and observing its motion by interference methods, a given amount of applied energy must produce a very large increase in the amplitude. In this manner it seems probable that frequencies of vibration of solids may be observed and measured which are beyond the limits of the human ear.

The authors are indebted to Brig. Gen. James Allen, Chief Signal Officer of the Army, and Dr. S. W. Stratton, Director of the Bureau of Standards, for facilities furnished.

WASHINGTON, September I, I9IO. 